\title{
Efficient expansion of human keratinocyte stem/progenitor cells carrying a transgene with lentiviral vector
}

Daisuke Nanba ${ }^{1,2^{*}}$, Natsuki Matsushita ${ }^{3}$, Fujio Toki ${ }^{1,2}$ and Shigeki Higashiyama ${ }^{2,4}$

\begin{abstract}
Introduction: The development of an appropriate procedure for lentiviral gene transduction into keratinocyte stem cells is crucial for stem cell biology and regenerative medicine for genetic disorders of the skin. However, there is little information available on the efficiency of lentiviral transduction into human keratinocyte stem/progenitor cells and the effects of gene transduction procedures on growth potential of the stem cells by systematic assessment.
\end{abstract}

Methods: In this study, we explored the conditions for efficient expansion of human keratinocyte stem/progenitor cells carrying a transgene with a lentiviral vector, by using the culture of keratinocytes on a feeder layer of 3 T3 mouse fibroblasts. The gene transduction and expansion of keratinocytes carrying a transgene were analyzed by Western blotting, quantitative PCR, and flow cytometry.

Results: Polybrene (hexadiamine bromide) markedly enhanced the efficiency of lentiviral gene transduction, but negatively affected the maintenance of the keratinocyte stem/progenitor cells at a concentration higher than $5 \mu \mathrm{g} / \mathrm{ml}$. Rho-assiciated kinase (ROCK) inhibitor Y-27632, a small molecule which enhanced keratinocyte proliferation, significantly interfered with the lentiviral transduction into cultured human keratinocytes. However, a suitable combination of polybrene and Y-27632 effectively expanded keratinocytes carrying a transgene.

Conclusions: This study provides information for effective expansion of cultured human keratinocyte stem/progenitor cells carrying a transgene. This point is particularly significant for the application of genetically modified keratinocyte stem/progenitor stem cells in regenerative medicine.

\section{Introduction}

Recombinant retroviruses enable a transgene to permanently integrate into the genome of a target cell, and are widely used as a vector in gene therapy [1,2]. Efficient and safe gene transduction into stem cells with viral vectors is indispensable for successful regenerative medicine, including the generation of genetically modified tissue stem cells and induced pluripotent stem (iPS) cells derived from patients affected by genetic disorders $[3,4]$. Transduction and silencing of genes with viral vectors are also essential for analyzing stem cell function during development, homeostasis and tumorigenesis. Thus, the

\footnotetext{
* Correspondence: nanba.daisuke.mk@ehime-u.ac.jp

'Senior Research Fellow Center, Ehime University, Shitsukawa, Toon, Ehime

791-0295, Japan

${ }^{2}$ Division of Cell Growth and Tumor Regulation, Proteo-Science Center,

Ehime University, Shitsukawa, Toon, Ehime 791-0295, Japan

Full list of author information is available at the end of the article
}

development of highly efficient and less-cytotoxic viral gene transduction into stem cells is crucial for advances in stem cell-based regenerative medicine and stem cell biology.

Human skin contains keratinocyte stem cells that are clonogenic when cultivated on a feeder layer of mouse 3 T3 fibroblasts, and show significant proliferative capacity in culture [5]. Ex vivo maintenance and expansion of human keratinocyte stem cells have achieved the autologous transplantation of confluent sheets of cultured keratinocytes onto patients with extensive burns [6]. The culture of keratinocyte stem cells has also enabled a gene therapy for a genetic disorder of the skin. De Luca and his colleagues [7] isolated epidermal keratinocyte stem cells from a patient who carries a null allele and a single point mutation in the $L A M B 3$ gene encoding laminin beta 3 subunit, and thus is affected by junctional epidermolysis bullosa. They transduced the full-length 
$L A M B 3$ cDNA into the keratinocyte stem cells with a retroviral vector, and prepared genetically corrected cultured epidermal grafts. The grafts were engrafted and remained stable for at least one year in the absence of blisters, infections, inflammations or immune response. Thus, gene transduction into cultured keratinocyte stem cells assures the healing or alleviation of inherent genetic disorders of the skin.

Polybrene (hexadimethrine bromide), a cationic polymer, has been widely used to increase the efficiency of retroviral transduction [8]. Cationic polymers, including polybrene, enhance virus adsorption on the surface of the cell by neutralizing the negative electrostatic repulsion between the cell surface and the virus particles $[9,10]$. It has been reported that polybrene is utilized for introduction of DNA into keratinocytes as a DNA carrier [11,12], and that it also enhances gene transduction into keratinocytes with adenoviruses [13,14] and retroviruses $[15,16]$. Besides polybrene, van den Bogaard et al. have recently reported that Rho-associated kinase (ROCK) inhibitor Y-27632 enhances lentiviral transduction into human keratinocytes [17]. However, there is little information available on how polybrene and Y-27632 impact the efficiency of lentiviral transduction into human keratinocytes, keratinocyte proliferation and maintenance of the stem/progenitor cells. Here, we provide information for an efficient and less-cytotoxic gene transduction with a lentiviral vector into human epidermal keratinocyte stem/progenitor cells cultured on a feeder layer of 3 T3 mouse fibroblasts by using polybrene and Y-27632.

\section{Methods}

\section{Cell culture}

The culture of normal human epidermal keratinocytes (strain no. 685389; KURABO, Osaka, Japan) isolated from the foreskin of newborns was previously described [18]. Briefly, frozen keratinocytes were thawed and cultivated at clonal density on a feeder layer of mitomycin C-treated $3 \mathrm{~T} 3-\mathrm{J} 2$ cells at $37^{\circ} \mathrm{C}$ as described $[19,20]$. The medium was changed every four days. Cells were used between passage 4 and 10. For determination of colonyforming efficiency, $1 \times 10^{4}$ keratinocytes were cultured as described above. Cultures were maintained for eight days, and subsequently fixed in $3.7 \%$ buffered formaldehyde, and stained with $1 \%$ rhodamine B (Sigma-Aldrich, St. Louis, MO, USA).

\section{Preparation of lentiviral vector}

A plasmid for lentiviral expression of enhanced green fluorescent protein (EGFP) was generated by subcloning the EGFP cDNA from pEGFP-N1 (Clontech, Palo Alto, CA, USA) into the HIV-based self-inactivating lentiviral expression vector plasmid pCSII-CMV-MCS [21]
(RIKEN BioResource Center, Tsukuba, Japan). The EGFP expression cassette under the control of CMV promoter was introduced upstream of woodchuck hepatitis virus posttranscriptional regulatory element in the transfer plasmid pCSII-CMV-MCS. Lentiviral vector pseudotyped with vesicular stomatitis virus glycoprotein was generated by standard DNA transfection. HEK293T cells were transfected with transfer, envelope and packaging plasmids using lipofectamine LTX (Invitrogen, Carlsbad, CA, USA). Viral vector particles were ultracentrifuged at 100,000 $\times$ g for $1 \mathrm{hr}$, and resuspended in Dulbecco-modified Eagle's medium. To measure the functional titer, HEK293T cells were seeded into a 24-well culture plate, transduced with proper concentrations of viral vectors, and the functional titer was analyzed by flow cytometry (FACS Calibur; Becton Dickinson, Franklin Lakes, NJ, USA). The multiplicity of infection (MOI) of 1 was defined as a number of functional lentiviral particles which was sufficient to introduce a transgene into HEK 293 cells with 100\% efficiency.

\section{Lentiviral infection}

A total of $10^{4}$ keratinocytes were seeded in a 12-well cell culture plate with mitomycin C-treated 3 T3-J2 cells. Keratinocytes were further incubated for four hours and then infected by replacing the infection medium containing lentiviral particles, polybrene and Y-27632 at various concentrations. The infection medium was removed and replaced by fresh medium after overnight incubation. The treatment with Y-27632 continued until Day 4 in culture. After seven days of cultivation, the expression level of the transgene was analyzed by Western blotting and flow cytometry. Polybrene (hexadiamine bromide) and Y-27632 were purchased from SigmaAldrich and Wako (Osaka, Japan), respectively. The procedure of lentiviral infection and subsequent analysis of gene transduction are described in Figure 1.

\section{Western blotting}

A total of $10^{4}$ keratinocytes were seeded in a 12-well cell culture plate with mitomycin C-treated 3 T3-J2 cells, grown for seven days, and analyzed by Western blotting, as previously described [18]. The luminescence signals were detected by using ImageQuant LAS4010 (GE Healthcare, Buckinghamshire, UK). Primary antibodies used were as follows: rabbit polyclonal antibody to GFP (\#598; 1:1,000; Medical \& Biological Laboratories, Nagoya, Japan), and mouse monoclonal antibody to $\alpha$-tubulin (\#T6199; 1:5,000; Sigma-Aldrich). Secondary antibodies were horseradish peroxidase (HRP)-conjugated goat antimouse and rabbit IgG (\#115-055-174 and \#211-032-171; 1:5,000; Jackson ImmunoResearch, West Grove, PA, USA). Relative density of EGFP bands was measured with Volocity (PerkinElmer, Branchburg, NJ, USA) and normalized with density of $\alpha$-tubulin bands. The values 


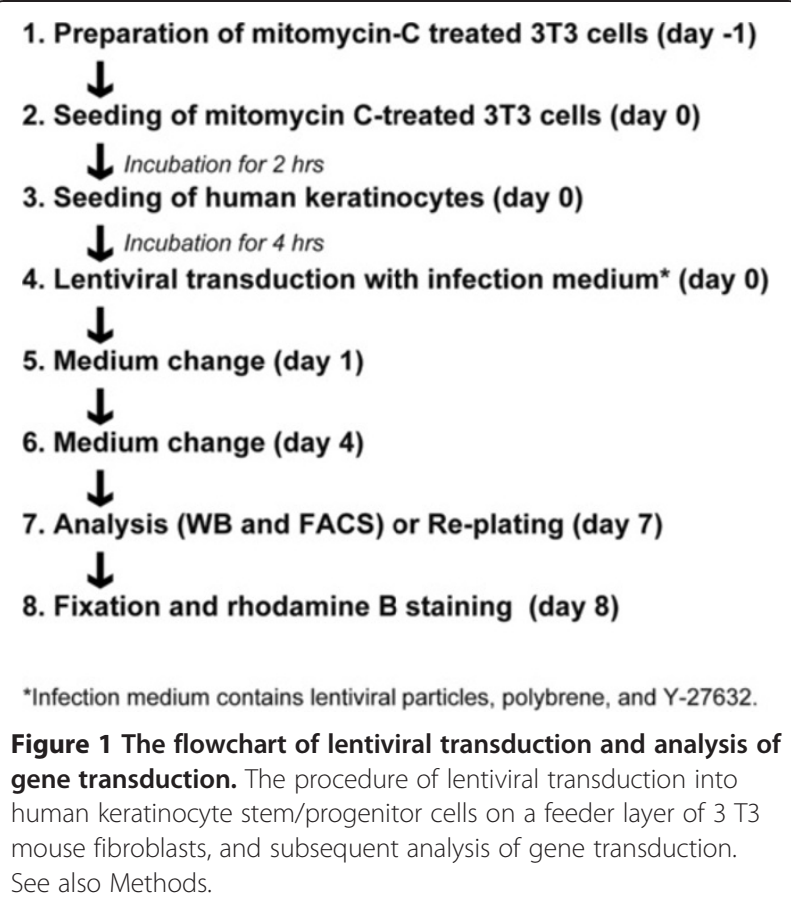

*Infection medium contains lentiviral particles, polybrene, and Y-27632.

Figure 1 The flowchart of lentiviral transduction and analysis of gene transduction. The procedure of lentiviral transduction into human keratinocyte stem/progenitor cells on a feeder layer of 3 T3 mouse fibroblasts, and subsequent analysis of gene transduction. See also Methods.

(means $\pm \mathrm{SD}$ ) were determined based on results in three independent experiments.

\section{Cell proliferation assay}

A total of $10^{4}$ keratinocytes were seeded in a 12 -well cell culture plate with mitomycin C-treated 3 T3-J2 cells. After seven days of cultivation, keratinocytes were trypsinized and stained with $0.4 \%$ Trypan blue (Invitrogen) to identify dead cells. Then, the number of cells was counted with a Countess ${ }^{\mathrm{TM}}$ automated cell counter (Invitrogen), which could eliminate the dead cells and large mitomycin C-treated 3 T3-J2 fibroblasts with irregular shape. The values (means $\pm \mathrm{SD}$ ) were determined based on results from three independent experiments.

\section{Immunofluorescence microscopy}

A total of $2 \times 10^{3}$ keratinocytes were seeded at clonal density in a $35-\mathrm{mm}$ cell culture dish with mitomycin C-treated 3 T3-J2 cells, grown for six days, and analyzed by immunofluorescence microscopy, as previously described [18]. Rat monoclonal antibody to $\alpha 6$ integrin (clone GoH3; 1:200; Becton Dickinson), and mouse monoclonal antibody to p63 (clone 4A4; 1:500; Millipore, Bedford, MA, USA) and involucrin (clone SY5; 1:1,000; Abcam, Cambridge, UK) were used as primary antibodies. Cy3-conjugated goat anti-rat IgG and anti-mouse IgG (\#112-165-167 and \#715-025-140; 1:500; Jackson ImmunoResearch) were used as secondary antibodies.

\section{Flow cytometry}

A total of $2 \times 10^{5}$ keratinocytes were seeded in a $60-\mathrm{mm}$ cell culture dish with mitomycin C-treated 3 T3-J2 cells, transduced EGFP cDNA with lentivirus vector in presence or absence of Y-27632, and grown for seven days. Trypsinized and resuspended keratinocytes were incubated with mouse monoclonal antibody against $\alpha 6$ integrin (clone 17D11; 1:20; a gift from Dr. Hirako, Nagoya University, Nagoya, Japan) for $1 \mathrm{hr}$ on ice. Keratinocytes were incubated with phycoerythrin (PE)-conjugated goat polyclonal antibody against mouse IgG (sc-3738; 1:250; Santa Cruz Biotechnology, Santa Cruz, CA, USA) for $1 \mathrm{hr}$ on ice. After three washes with Hank's Balanced salt solution containing calcium and magnesium (HBSS $(+)$ ), the cells were then resuspended in $\operatorname{HBSS}(+)$ and analyzed by using the FACSCan flow cytometer (Becton Dickinson).

\section{Calculation of EGFP-positive keratinocytes and 3 T3 feeder cells}

The percentages of EGFP-positive keratinocytes and 3 T3 feeder cells were calculated by the following equations: EGFP-positive keratinocytes $(\%)=(\%$ of EGFPpositive and ITGA6-positive cells)/(\% of EGFP-positive and ITGA6-positive cells $+\%$ of EGFP-negative and ITGA6-positive cells) $\times 100$, and EGFP-positive 3 T3 feeder cells $(\%)=(\%$ of EGFP-positive and ITGA6negative cells)/(\% of EGFP-positive and ITGA6-negative cells $+\%$ of EGFP-negative and ITGA6-negative cells) $\times$ 100 . The values (means \pm SD) were determined based on results from three independent experiments.

\section{Quantitative PCR}

A total of $2 \times 10^{5}$ keratinocytes were seeded in a $60 \mathrm{~mm}$ cell culture dish with mitomycin C-treated 3 T3-J2 cells, transduced EGFP cDNA with lentivirus vector in the presence or absence of Y-27632, and grown for seven days. Genomic DNA was extracted from keratinocytes and 3 T3-J2 cells with lysis buffer consisting of $50 \mathrm{mM}$ Tris-HCl (pH 8.0), $100 \mathrm{mM} \mathrm{NaCl}, 20 \mathrm{mM}$ EDTA, 1\% SDS, and 5 units $/ \mathrm{mL}$ of Proteinase K (Sigma-Aldrich). The DNA (50 ng) was subjected to quantitative PCR using Power SYBR Green PCR Master Mix Reagents and a 7300 Real-Time PCR System (Applied Biosystems, Foster City, CA, USA). Relative copy number of the transgene was calculated based on the $\Delta \Delta \mathrm{Ct}$ method by normalization using the $\mathrm{Ct}$-value of the human or mouse phosphoglycerate kinase $(P G K)$ gene. The detection primers used were as follows: 5'-GTGAACGGATCTA CAAATGGCAG and 5'- GTCTGTTGCTATTATGTC TACTA for the recombinant lentiviral vector, $5^{\prime}$ - TGA TTATTGGTGGTGGAATGGCTT and 5'-TGGAGGT CAGCATCTATACTAAGA for the human PGK gene, and 5 '-TGCTAGACAAAGTCAATGAGATGA and 5'- 
TGATATGCAACCACTGTGAAAGGGT for the mouse $P G K$ gene. The values (means $\pm \mathrm{SD}$ ) were determined based on results from three independent experiments.

\section{Results}

Polybrene enhances transduction efficiency but reduces growth potential of keratinocytes

To evaluate gene transduction with a lentiviral vector, we prepared a lentiviral vector expressing EGFP under the control of the cytomegalovirus (CMV) promoter. After four hours of seeding on a feeder layer of mitomycin C-treated $3 \mathrm{~T} 3$ cells, keratinocytes were infected with the EGFP-expressing virus at various MOI, and the expression of EGFP was analyzed by Western blotting after seven days of culture (Figure 1). Increased expression levels of EGFP correlated with the increase in the MOI (Figure 2A). Less than $10 \%$ of keratinocytes expressed EGFP at MOI 1 (Figure 2C), a condition in which almost
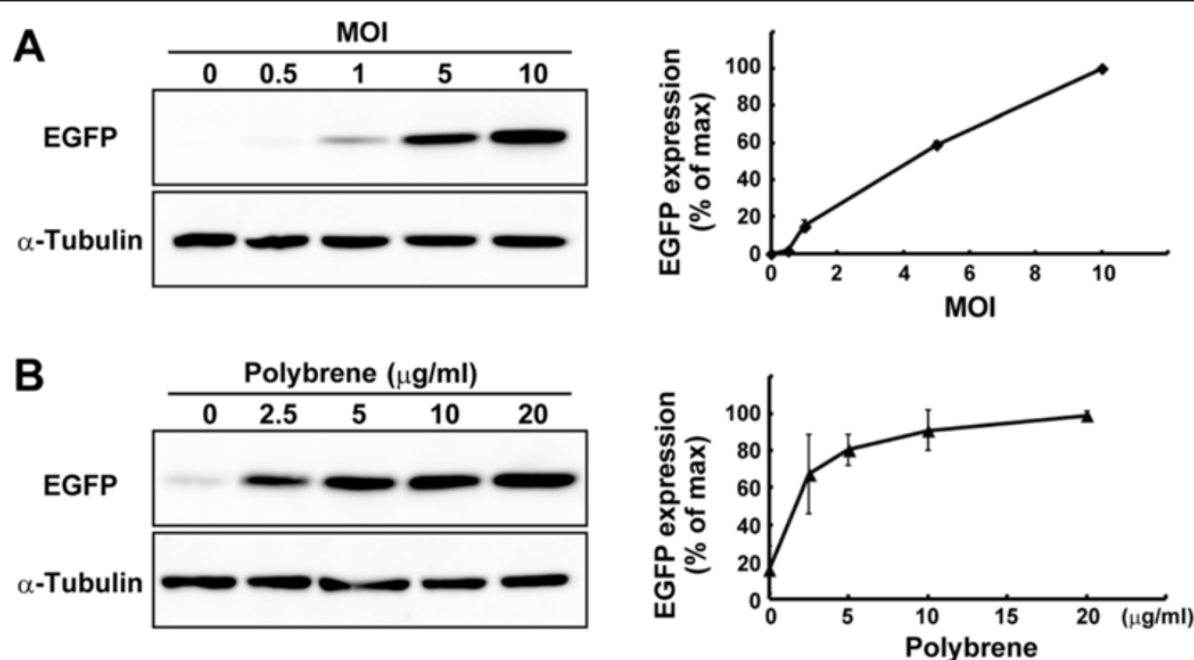

C Polybrene $(\mu \mathrm{g} / \mathrm{ml})$

D
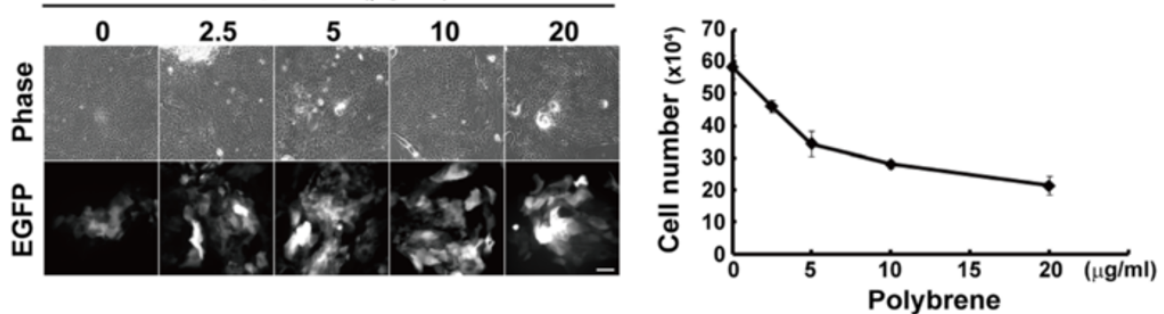

$\mathbf{E}$

Polybrene $(\mu \mathrm{g} / \mathrm{ml})$

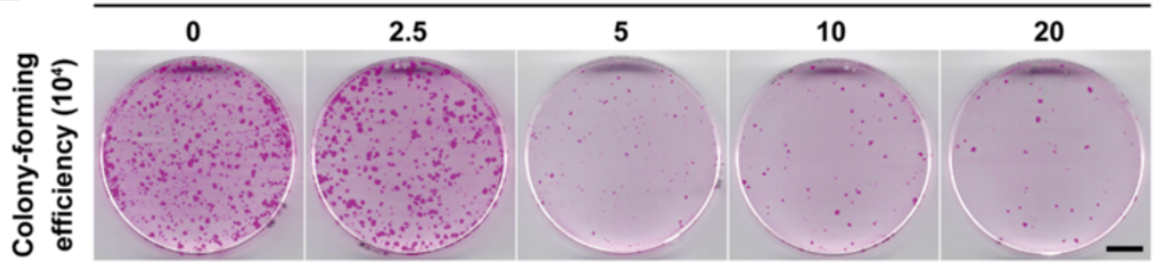

Figure 2 The effects of polybrene on gene transduction efficiency and growth potential of keratinocytes. (A) The left panel shows Western blotting of lysates from keratinocytes transduced with the enhanced green fluorescent protein (EGFP)-expressing lentivirus at various multiplicity of infection (MOI). The expression of a-tubulin was used as loading control. Right panel shows relative expression levels of EGFP. The values (mean \pm SD) were obtained from the density of bands normalized with a-tubulin bands. (B) The left panel shows Western blotting of lysates from keratinocytes transduced with the EGFP-expressing lentivirus at MOI 1, and various concentration of polybrene. The expression of a-tubulin was used as loading control. Right panel shows relative expression levels of EGFP. The values (mean \pm SD) were obtained from the density of bands normalized with a-tubulin bands. (C) Images of phase-contrast and EGFP expression in keratinocytes transduced with the EGFP-expressing lentivirus at MOI 1, and various concentrations of polybrene. Bar, $100 \mu \mathrm{m}$. (D) The effects of polybrene on keratinocyte proliferation are shown. Keratinocytes $\left(10^{4}\right.$ cells) were cultivated with various concentration of polybrene during lentivirus transduction, and the number of keratinocytes were counted after seven days of infection. (E) Determination of colony-forming efficiency (CFE) of keratinocytes infected with lentiviral particles at $\mathrm{MOI} 1$, and various concentrations of polybrene. After seven days of infection, $10^{4}$ cells were passaged and cultured without polybrene for eight days until the cultures were fixed and stained with rhodamine B. Bar, $10 \mathrm{~mm}$. The values (means \pm SD) in A, $B$, and $D$ were determined based on results from triplicate experiments. 
all HEK293 cells expressed EGFP. We examined the effects of polybrene on lentiviral gene transduction at MOI 1 into human epidermal keratinocytes. Polybrene was added into the medium in which keratinocytes were incubated overnight with lentiviral particles. The next day, the medium containing polybrene and the lentiviruses was removed and replaced with fresh medium. Keratinocytes were further cultivated for six days, and the expression of EGFP was analyzed by Western blotting. As the concentration of polybrene increased, the expression levels of EGFP also increased (Figure 2B, C). An overnight treatment with polybrene for lentiviral transduction, however, inhibited the proliferation of keratinocytes, even if keratinocytes were treated with only $2.5 \mu \mathrm{g} / \mathrm{ml}$ of polybrene (Figure 2D). We further investigated the effect of polybrene on the growth potential of keratinocytes. Keratinocytes were treated with polybrene overnight for lentiviral transduction at MOI 1, and the same number of keratinocytes $\left(10^{4}\right.$ cells) was passaged into a new $6-\mathrm{cm}$ cell culture dish after seven days of culture. The keratinocytes were then maintained without polybrene for eight days until the culture was fixed and stained with rhodamine $\mathrm{B}$. The results indicated that transient treatment of keratinocytes with polybrene negatively affected the maintenance of growth potential of human keratinocytes at a concentration higher than $5 \mu \mathrm{g} / \mathrm{ml}$ (Figure 2E). Collectively, the treatment of keratinocytes with $2.5 \mu \mathrm{g} / \mathrm{ml}$ of polybrene could enhance lentiviral transduction efficiency significantly and maintain their growth potential.

We next examined whether negative effects of polybrene on keratinocyte proliferation and growth potential resulted from the toxicity of EGFP expression in transduced keratinocytes. An overnight treatment with polybrene after seeding markedly inhibited the proliferation of untransduced keratinocytes (Figure 3A), as shown in transduced keratinocytes (Figure 2D). Furthermore, as the concentration of polybrene increased, the colonyforming efficiency (CFE) decreased (Figure 3B). These data confirmed that polybrene itself negatively impacted the proliferation and maintenance of growth potential of human keratinocytes regardless of the EGFP expression.

\section{Clonal expansion of keratinocyte stem/progenitor cells expressing a transgene}

Keratinocytes were seeded at clonal density, and transduced with the EGFP-expressing lentivirus. In a condition in which lentiviruses were infected into keratinocytes at MOI 1 in the presence of $2.5 \mu \mathrm{g} / \mathrm{ml}$ of polybrene, progressively growing colonies expressed EGFP in almost all cells (Figure 4A). Keratinocytes could be identified as $\alpha 6$ integrin (ITGA6)-expressing cells [22] (Figure 4B). These growing colonies are formed by keratinocyte stem/progenitor cells [18]. We confirmed that EGFP-expressing growing

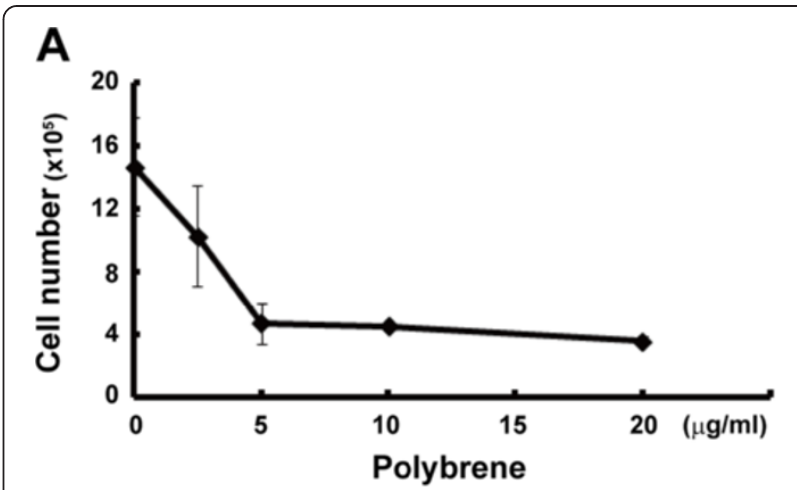

B

Polybrene $(\mu \mathrm{g} / \mathrm{ml})$

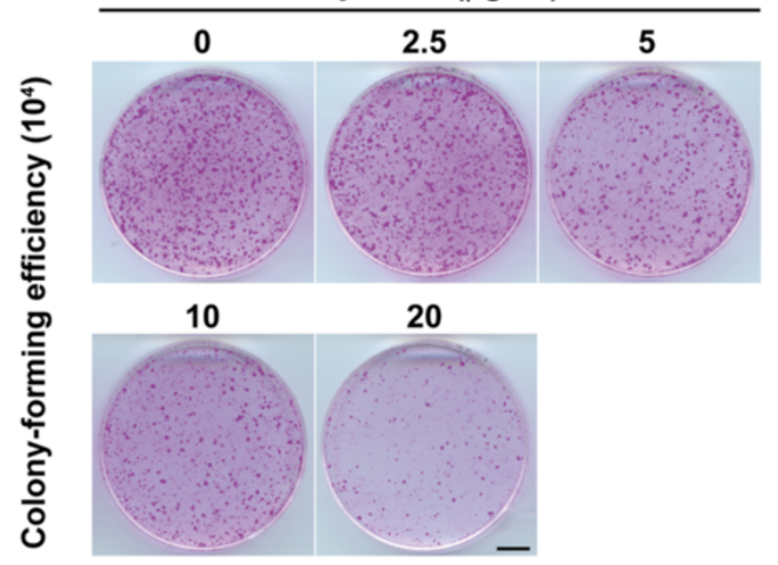

Figure 3 Polybrene negatively impacts the proliferation and maintenance of growth potential of human keratinocytes.

(A) The effects of polybrene on keratinocyte proliferation are shown. Keratinocytes $\left(2 \times 10^{5}\right.$ cells) were seeded and treated with various concentration of polybrene overnight, and then the number of keratinocytes were counted after seven days of cultivation. The values (means \pm SD) were determined based on results in triplicate experiments. (B) Determination of colony-forming efficiency (CFE) of keratinocytes cultivated with various concentrations of polybrene is shown. After seven days of cultivation with the condition described above, $10^{4}$ cells were passaged and cultured without polybrene for eight days until the cultures were fixed and stained with rhodamine B. Bar, $10 \mathrm{~mm}$.

colonies expressed p63, a transcriptional factor that is expressed in keratinocyte stem cells [23], and is essential for significant growth capacity of keratinocytes [24] (Figure 4C). EGFP-expressing growing colonies also possessed the ability to stratify and express a terminal differentiation marker, involucrin (INV) [25] (Figure 4D). The expressions of these marker proteins in the colony derived from EGFP-expressing keratinocyte stem/progenitor cells were completely identical to those in progressively growing colonies formed by untransduced keratinocyte stem/ progenitor cells (Figure 4E). We further confirmed the long-term maintenance of EGFP expression in keratinocytes after cloning of an EGFP-expressing keratinocyte colony (Figure 4F). These results indicated that 


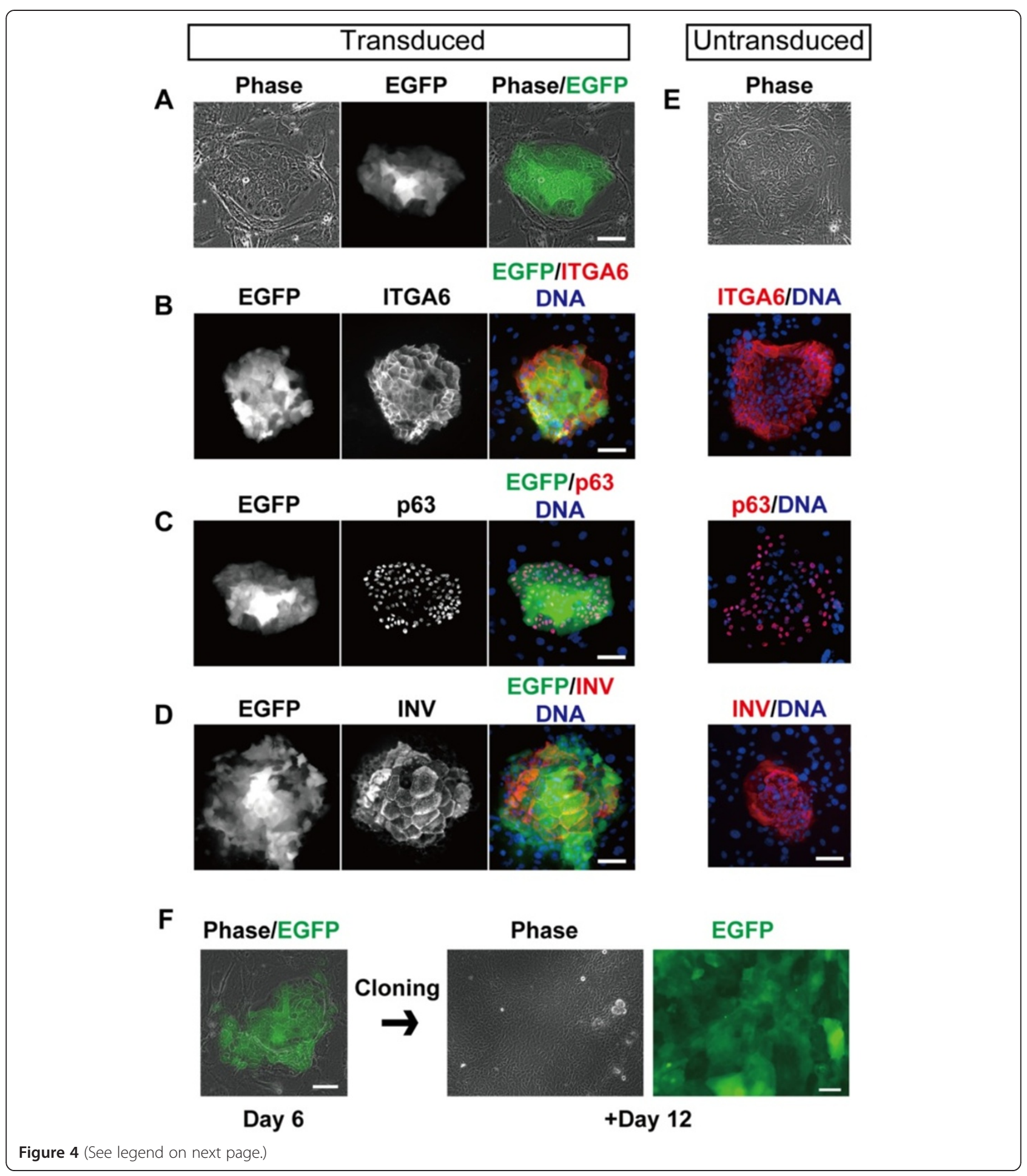




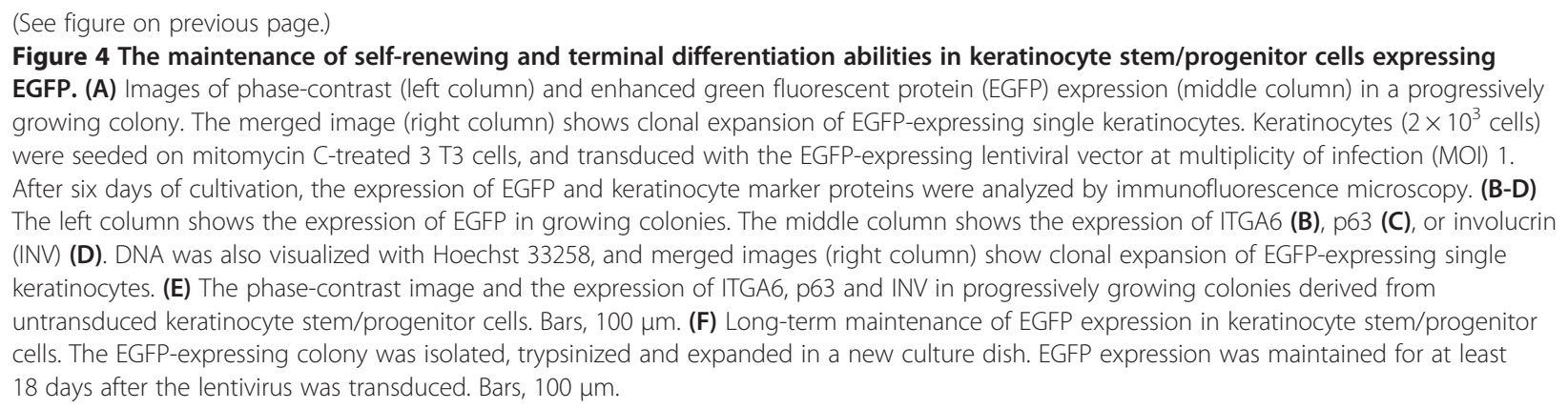

keratinocyte stem/progenitor cells carrying a transgene could be maintained long-term in the presence of $2.5 \mu \mathrm{g} / \mathrm{ml}$ of polybrene during lentiviral transduction.

\section{Lower transduction efficiency of 3 T3 feeder cells}

Lentiviral transduction enables a transgene to permanently integrate into the genome of cells, even if the cells do not proliferate [26]. However, the expression of EGFP was mainly observed in keratinocyte colonies, but not in the feeder cells, after seven days of transduction as shown above (Figure 4A-D). The lower transduction efficiency of 3 T3 feeder cells favors the generation and transplantation of genetically engineered keratinocyte stem/progenitor cells because transduction of a gene that functions in keratinocytes into the 3 T3 feeder cells might cause unexpected effects. To confirm the difference in transduction efficiency between keratinocytes and mitomycin C-treated 3 T3 fibroblasts precisely, we evaluated the percentage of EGFP-expressing cells in both cell types by flow cytometry. Keratinocytes were recognized as ITGA6-positive cells in a mixed cell population containing keratinocytes and fibroblasts [27]. In a condition in which lentiviruses were infected into keratinocytes at MOI 1 in the presence of $2.5 \mu \mathrm{g} / \mathrm{ml}$ polybrene, $23.4 \pm 2.62$ (mean $\pm \mathrm{SD}$ )\% of ITGA6-positive keratinocytes expressed EGFP (Figure 5A and See Methods). However, the expression of EGFP was only detectable in $1.64 \pm 1.71$ (mean $\pm \mathrm{SD}$ )\% of ITGA6-negative 3 T3 fibroblasts (Figure 5A and See Methods).

\section{ROCK inhibitor interferes with lentiviral transduction into keratinocytes}

The addition of the ROCK inhibitor Y-27632 into the culture medium results in the increased proliferation of human keratinocytes [28-30]. The mechanism is still unknown; however, it could have investigational and medical applications. Therefore, we examined the effect of Y-27632 on lentiviral transduction into human keratinocytes. Lentiviruses encoding EGFP cDNA were infected with or without $10 \mu \mathrm{M}$ Y-27632, and the efficiency of gene transduction was analyzed by flow cytometry after seven days of cultivation (Figure 5A-D). In a condition in which lentiviruses were infected into keratinocytes at MOI 1 in the presence of $2.5 \mu \mathrm{g} / \mathrm{ml}$ polybrene, $23.4 \pm 2.62 \%$ (mean $\pm \mathrm{SD}$ ) of ITGA6-positive keratinocytes expressed EGFP (Figure 5A and See Methods). However, only $8.51 \pm 1.68 \%$ (mean \pm SD) of ITGA6-positive keratinocytes cultured in the presence of $10 \mu \mathrm{M}$ Y-27632 expressed EGFP (Figure 5B and See Methods). We further examined the integration of the transgene gene into the genome of keratinocytes and 3 T3 fibroblasts, with or without Y-27632. Quantitative PCR revealed that the integration of the exogenous EGFP gene into keratinocytes was significantly interfered with the treatment of Y-27632 (Figure 5E). This analysis also confirmed that EGFP-expressing lentiviruses easily infected into keratinocytes, compared to 3 T3 fibroblasts (Figure 5A), as shown in Figure 3. Interestingly, Y-27632 inhibited lentiviral transduction into keratinocytes, but not into a feeder layer of 3 T3 fibroblasts (Figure 5B, E). We also counted the number of EGFP-positive cells in the keratinocyte cultures two days after lentiviral transduction with or without $10 \mu \mathrm{M}$ Y-27632, and confirmed that Y-27632 inhibited lentiviral transduction (Figure 5F). This result indicated that the decreased ratio of EGFPexpressing keratinocytes with Y-27632 after seven days of cultivation was not mainly due to the increased expansion of untransduced keratinocytes with Y-27632.

\section{A combination of polybrene and Y-27632 efficiently expands keratinocytes carrying a transgene}

We confirmed that Y-27632 increased colony-forming efficiency and keratinocyte proliferation, even if used at $1.0 \mu \mathrm{M}$ (Figure 6A). Finally, we explored the condition in which keratinocytes carrying EGFP transgene were expanded the most efficiently. EGFP-expressing lentiviruses were infected into keratinocytes at MOI 1 in the presence of $2.5 \mu \mathrm{g} / \mathrm{ml}$ polybrene and various concentrations of Y-27632. Keratinocytes were treated with Y-27632 for four days after the first day of cultivation. We evaluated the efficiency of gene transduction into keratinocytes by flow cytometry after seven days of cultivation. Although the number of keratinocytes increased (Figure 6B), the percentage of EGFP-positive keratinocytes 


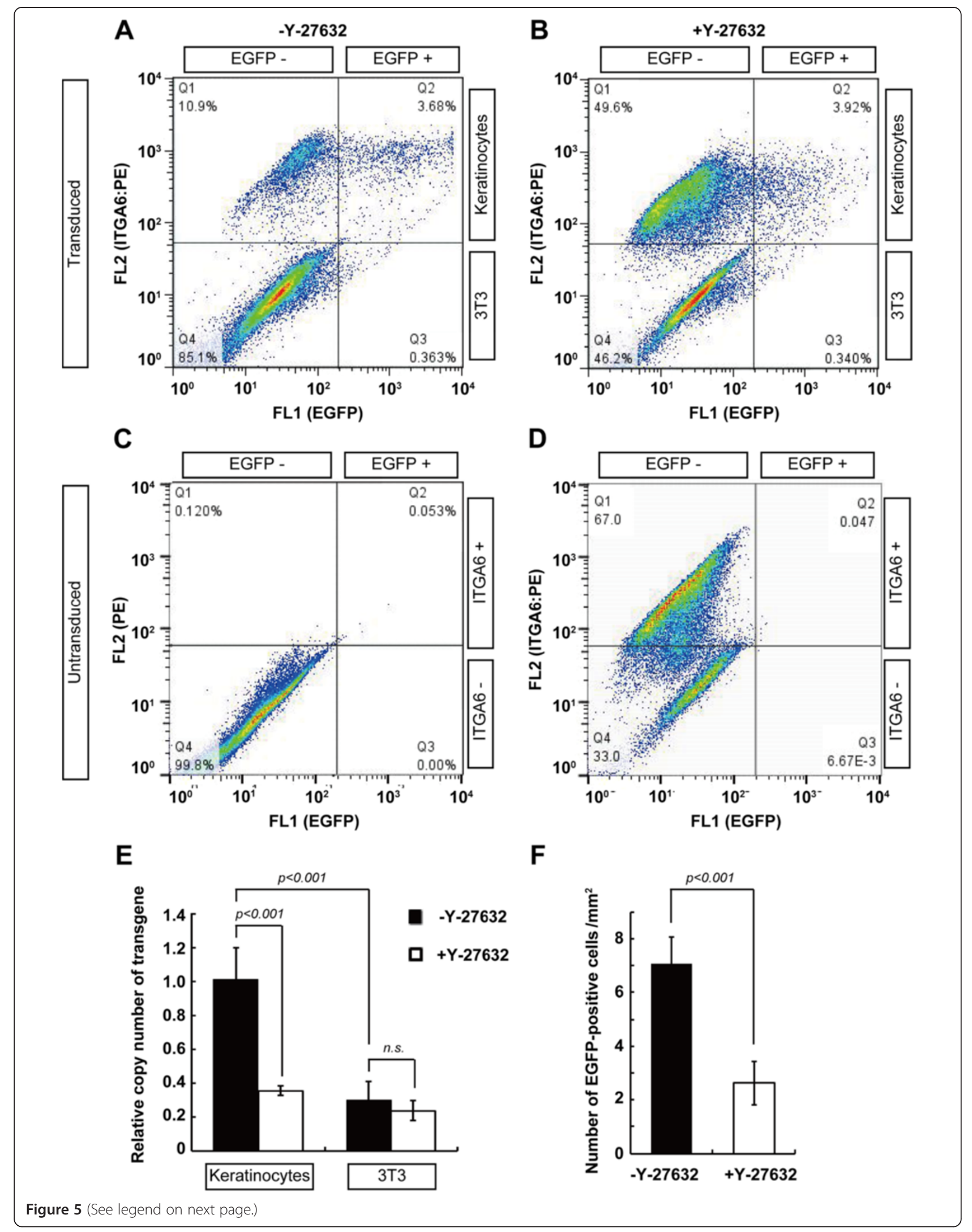


(See figure on previous page.)

Figure 5 The interference of a ROCK inhibitor with lentiviral gene transduction. (A-D) Flow cytometric analysis of keratinocyte culture on a feeder layer of 3 T3 fibroblasts in the presence (B) and absence (A) of Rho-associated kinase (ROCK) inhibitor Y-27632. Keratinocytes $\left(5 \times 10^{5}\right.$ cells) were seeded on mitomycin C-treated 3 T3 cells, and transduced with the enhanced green fluorescent protein (EGFP)-expressing lentiviral vector at multiplicity of infections (MOI) 1, with and without $10 \mu \mathrm{M}$ of Y-27632. After seven days of cultivation, the expression levels of EGFP and ITGA6 were analyzed by flow cytometry. ITGA6-positive and -negative cells were recognized as keratinocytes and 3 T3 fibroblasts, respectively. Representative dot plot images of triplicate experiments in each experimental condition are shown. Numerical values indicate the percentage of cells in each subpopulation. (C-D) Negative controls of flow cytometric analysis of keratinocyte culture on a feeder layer of 3 T3 fibroblasts shown in Figure 3. Keratinocytes $\left(5 \times 10^{5}\right.$ cells) were seeded on mitomycin C-treated 3 T3 cells, grown for seven days, and analyzed by flow cytometry. Flow cytometric analysis of the untransduced suspended keratinocytes and 3 T3 fibroblasts incubated with only PE-conjugated secondary antibodies (C), or with PE-conjugated secondary antibodies after treatment with anti-ITGA6 antibodies (D). Numerical values indicate the percentage of cells in each subpopulation. (E) Quantitative PCR analysis of relative copy number of EGFP transgene in keratinocytes and 3 T3 fibroblasts in the presence ( $\square$ ) and absence ( $\mathbf{a})$ of $10 \mu \mathrm{M}$ of $\mathrm{Y}$-27632. (F) The number of EGFP-positive cells in the keratinocyte cultures after two days of lentiviral transduction in the presence and absence of $10 \mu \mathrm{M}$ of Y-27632. P-value was calculated by Student's t-test. n.s., not significant. The values (means $\pm \mathrm{SD}$ ) in $\mathrm{E}$ and $\mathrm{F}$ were determined based on results from triplicate experiments.

decreased as the concentration of Y-27632 increased (Figure 6C and See Methods). However, the net number of EGFP-expressing keratinocytes increased as the concentration of Y-27632 increased (Figure 6D), and still did not reach a plateau by treatment with $20 \mu \mathrm{M}$ of Y-27632 (Figure 6D). The effects of higher concentrations of Y-27632 should be tested. These data clearly indicated that a combination of $2.5 \mu \mathrm{g} / \mathrm{ml}$ polybrene and Y-27632 effectively expanded keratinocytes carrying EGFP transgene.

\section{Discussion}

Gene transduction with lentiviral vectors has already been used for basic and clinical studies of human keratinocytes.
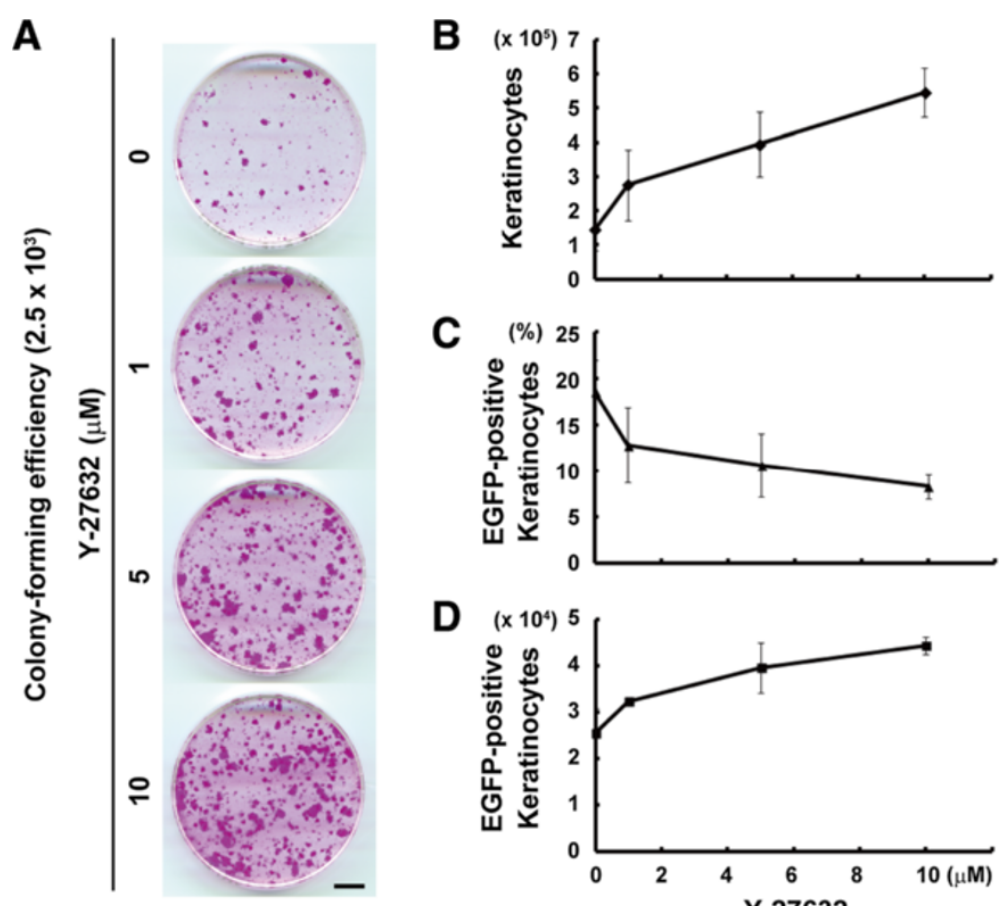

D

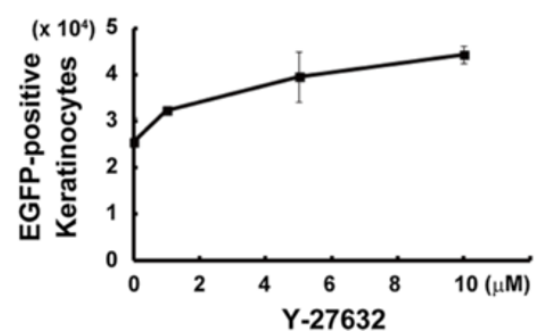

Figure 6 Efficient expansion of keratinocytes carrying EGFP with a combination of polybrene and Y-27632. (A) Determination of colony-forming efficiency of keratinocytes treated with various concentrations of Y-27632 for four days, after the first day of cultivation. A total of $2.5 \times 10^{3}$ keratinocytes were seeded and cultured for 12 days until the cultures were fixed and stained with rhodamine B. Bar, $10 \mathrm{~mm}$. (B-D) Flow cytometric analysis of keratinocytes cultured on a feeder layer of 3 T3 fibroblasts with various concentrations of Y-27632. Keratinocytes $\left(5 \times 10^{5}\right.$ cells) were cultivated with various concentrations of $\mathrm{Y}-27632$ during lentivirus transduction at multiplicity of infections (MOI) 1 , and then the keratinocyte cultures were analyzed after seven days of transduction. ITGA6-positive and -negative cells were recognized as keratinocytes and 3 T3 fibroblasts, respectively. The quantitative representation of the effects of Y-27632 on keratinocyte proliferation (B), transduction efficiency into the keratinocytes (C) and the expansion of enhanced green fluorescent protein (EGFP)-expressing keratinocytes (D). The values (means \pm SD) were determined based on results from triplicate experiments. 
In this study, we demonstrated the efficient expansion of human keratinocyte stem/progenitor cells carrying a transgene with lentiviral vector by using polybrene and Y-27632. This work has three major findings concerning lentiviral transduction into keratinocytes. First, polybrene markedly enhances the efficiency of lentiviral gene transduction, but reduces keratinocyte proliferation and negatively impacts the maintenance of keratinocyte stem/ progenitor cells at a concentration higher than $5 \mu \mathrm{g} / \mathrm{ml}$. Second, ROCK inhibitor Y-27632 significantly interferes with the lentiviral transduction into cultured human keratinocytes. Third, a suitable combination of polybrene and Y-27632 can effectively expand keratinocytes carrying a transgene.

Polybrene is widely used in viral gene transduction. Polybrene has no cytotoxic activity at low concentrations, but negatively affects the proliferation of corneal keratinocytes at concentrations greater than $10 \mu \mathrm{g} / \mathrm{ml}$ [31]. It also negatively impacts the proliferation of human mesenchymal stem cells, even if used at $1 \mu \mathrm{g} / \mathrm{ml}$ [32]. It has been reported that treatment with 2 to $6 \mu \mathrm{g} / \mathrm{ml}$ of polybrene results in the highest efficiency of gene transduction with adenoviruses in human epidermal keratinocytes [14]. In our experiments, $20 \mu \mathrm{g} / \mathrm{ml}$ of polybrene was required to induce the highest expression of transgene in lentiviral transduction. However, polybrene significantly reduced the proliferation of keratinocytes, even if used at low concentrations. Furthermore, we demonstrated that polybrene negatively impacted the maintenance of the keratinocyte stem/progenitor cells at a concentration higher than $5 \mu \mathrm{g} / \mathrm{ml}$. In serial culture, keratinocyte stem cells progressively lose their proliferative capacity to become transient amplifying cells with limited growth, a phenomenon termed clonal conversion [33]. Clonal conversion is accelerated by stress, suboptimal culture conditions, serial cultivation and age of donor [33]. It is also governed by the balance of Rac1 and Akt signaling activity and actin filament organization [18]. As polybrene modulates the charge of the cell surface, it might affect these intracellular signaling pathways and actin network, and then increase the rate of clonal conversion. Retroviral gene transfer into keratinocytes is also enhanced by another polycationic polymer, protamine sulfate [31] and by their cultivation on a substrate of fibronectin [16]. The effects of these reagents on transduction efficiency and clonal conversion should be further investigated.

The process of retroviral infection involves the absorption of viral particles on the host plasma membrane, the entry of the viral protein-RNA complexes into the cytoplasm, the reverse transcription of viral RNA into DNA and the intracellular trafficking and entry of viral protein-DNA complexes into the nucleus, which is followed by the integration of viral DNA into the host genome [26]. We have clearly demonstrated here that the ROCK inhibitor Y-27632 interfered with the lentiviral transduction in human epidermal keratinocytes. ROCK regulates both actin polymerization and phosphorylation of myosin regulatory light chain [34]. Recently, it has also been shown that ROCK regulates microtubule dynamics through phosphorylation of the tubulin polymerization promoting protein 1 (TPPPA/p25) [35]. The cytoskeleton, including actin filaments and microtubules in the host cells, contributes to intracellular transport of retroviral genomes from the cytoplasm into the nucleus [36-38]. As further investigations should be required, ROCK activity could be involved in intracellular trafficking of the retroviral genome.

Interestingly, van den Bogaard et al. have recently reported that Y-27632 enhances lentiviral transduction into human keratinocytes [17]. This report seems to be contrary to our results. However, van den Bogaard et al. have used high-passage adult keratinocytes for lentivirus transduction and the generation of human skin equivalents (HSEs), and then assessed the efficiency of lentiviral transduction into keratinocytes in HSEs, but not in cultured keratinocytes. Human adult keratinocytes significantly decrease their proliferative capacity by serial cultivation and stress in culture, including gene transduction [33]. Therefore, the enhanced expansion of EGFPexpressing adult keratinocytes in HSEs by Y-27632 might be due to the fact that the expansion of Y-27632-treated adult keratinocytes expressing EGFP is more efficient than that of untreated EGFP-expressing adult keratinocytes during the formation of HSEs.

van den Bogaard et al. has also reported high levels of GFP expression in the differentiated layers of HSEs [17]. We also observed that EGFP was strongly expressed in stratified and differentiating cells in the center of colonies. These results suggest that CMV promoter activity is enhanced in the stratified and differentiating keratinocytes. Y-27632 inhibits keratinocyte differentiation [28-30]. These observations alone suggest that Y-27632 inhibits EGFP expression under the control of the CMV promoter, but not lentiviral transduction itself. However, our quantitative PCR analysis has revealed that the integration of the exogenous EGFP gene into keratinocytes significantly interfered with the treatment of Y-27632. Consequently, reduced expression of EGFP after lentiviral transduction with Y-27632 might be, at least partially, due to the inhibition of keratinocyte differentiation and, therefore, CMV promoter activity by Y-27632, as well as lower transduction efficiency by the treatment with Y-27632.

We have demonstrated in this study that keratinocytes gave rise to colonies derived from their stem/progenitor cells carrying a transgene after lentiviral transduction with lower concentration of polybrene. We have also 
shown that the keratinocyte stem/progenitor cells carrying EGFP transgene could maintain both self-renewing and terminal differentiation abilities that are indispensable for making a functional epidermal sheet ex vivo, and permanent engraftment after transplantation $[33,39]$. These results indicate that a homologous clone of a keratinocyte stem cell carrying a transgene can be isolated and expanded massively for cell therapy for genetic disorders of the skin $[7,40]$.

\section{Conclusions}

A suitable combination of polybrene and Y-27632 efficiently expands keratinocyte stem/progenitor cells carrying a transgene, although polybrene and Y-27632 negatively affect growth and lentiviral transduction of human keratinocyte stem/progenitor cells, respectively. This point is particularly significant for the application of genetically modified keratinocyte stem/progenitor stem cells in regenerative medicine.

\section{Abbreviations}

CFE: Colony-forming efficiency; CMV: Cytomegalovirus; EGFP: Enhanced green fluorescent protein; HBSS (+): Hank's balanced salt solution containing calcium and magnesium; HRP: Horseradish peroxidase; HSEs: Human skin equivalents; INV: Involucrin; ITGA6: Integrin alpha 3; LAMB3: Laminin beta 3; MOl: Multiplicity of infection; PE: Phycoerythrin

\section{Competing interests}

The authors declare that they have no competing interests.

\section{Authors' contributions}

DN and NM designed the experiments. DN and FT performed cell culture experiments, Western blotting and flow cytometry. NM produced lentiviruses and performed $\mathrm{qPCR}$ analysis. DN and $\mathrm{SH}$ analyzed and interpreted the data. DN wrote the manuscript. All authors read and approved the final manuscript.

\section{Acknowledgements}

We thank Dr. Y. Hirako (Nagoya University, Japan) for providing us anti-a6 integrin antibody (clone 17D11), Dr. A.W. Amici (Ecole Polytechnique Fédérale de Lausanne, Switzerland) for critical reading of the manuscript, and Mrs. S. Matsushita for lentivirus production (Ehime University, Japan). We are also grateful to Japan Tissue Engineering Co., Ltd. (Gamagori, Japan) for providing us 3 T3-J2 cells. This work was supported by Grant-in Aid for Scientific Research from Japan Society for the Promotion of Science (No. 24791159) to D.N.

\section{Author details}

'Senior Research Fellow Center, Ehime University, Shitsukawa, Toon, Ehime 791-0295, Japan. 2Division of Cell Growth and Tumor Regulation, ProteoScience Center, Ehime University, Shitsukawa, Toon, Ehime 791-0295, Japan. ${ }^{3}$ Translational Research Center, Ehime University Hospital, Ehime University, Shitsukawa, Toon, Ehime 791-0295, Japan. ${ }^{4}$ Department of Biochemistry and Molecular Genetics, Graduate School of Medicine, Ehime University, Shitsukawa, Toon, Ehime 791-0295, Japan.

Received: 17 May 2013 Revised: 31 May 2013

Accepted: 14 October 2013 Published: 18 October 2013

\section{References}

1. Mulligan RC: The basic science of gene therapy. Science 1993, 260:926-932.

2. Andreadis ST, Roth CM, Le Doux JM, Morgan JR, Yarmush ML: Large-scale processing of recombinant retroviruses for gene therapy. Biotechnol Prog 1999, 15:1-11.
3. Passier R, van Laake LW, Mummery CL: Stem-cell-based therapy and lessons from the heart. Nature 2008, 453:322-329.

4. Izpisua Belmonte JC, Ellis J, Hochedlinger K, Yamanaka S: Induced pluripotent stem cells and reprogramming: seeing the science through the hype. Nat Rev Genet 2009, 10:878-883.

5. Barrandon $\mathrm{Y}$, Green $\mathrm{H}$ : Three clonal types of keratinocyte with different capacities for multiplication. Proc Natl Acad Sci USA 1987, 84:2302-2306.

6. Gallico GG 3rd, O'Connor NE, Compton CC, Kehinde O, Green H: Permanent coverage of large burn wounds with autologous cultured human epithelium. N Engl J Med 1984, 311:448-451.

7. Mavilio F, Pellegrini G, Ferrari S, Di Nunzio F, Di lorio E, Recchia A, Maruggi G, Ferrari G, Provasi E, Bonini C, Capurro S, Conti A, Magnoni C, Giannetti A, De Luca M: Correction of junctional epidermolysis bullosa by transplantation of genetically modified epidermal stem cells. Nat Med 2006, 12:1397-1402

8. Toyoshima K, Vogt PK: Enhancement and inhibition of avian sarcoma viruses by polycations and polyanions. Virology 1969, 38:414-426.

9. Coelen RJ, Jose DG, May JT: The effect of hexadimethrine bromide (polybrene) on the infection of the primate retroviruses SSV 1/SSAV 1 and BaEV. Arch Virol 1983, 75:307-311.

10. Davis HE, Morgan JR, Yarmush ML: Polybrene increases retrovirus gene transfer efficiency by enhancing receptor-independent virus adsorption on target cell membranes. Biophys Chem 2002, 97:159-172.

11. Staedel C, Remy JS, Hua Z, Broker TR, Chow LT, Behr JP: High-efficiency transfection of primary human keratinocytes with positively charged lipopolyamine:DNA complexes. J Invest Dermatol 1994, 102:768-772.

12. Nead MA, McCance DJ: Poly-L-ornithine-mediated transfection of human keratinocytes. J Invest Dermatol 1995, 105:668-671.

13. Jacobsen F, Hirsch T, Mittler D, Schulte M, Lehnhardt M, Druecke D, Homann HH, Steinau HU, Steinstraesser L: Polybrene improves transfection efficacy of recombinant replication-deficient adenovirus in cutaneous cells and burned skin. J Gene Med 2006, 8:138-146.

14. Doebis C, Ritter T, Brandt C, Schonberger B, Volk HD, Seifert M: Efficient in vitro transduction of epithelial cells and keratinocytes with improved adenoviral gene transfer for the application in skin tissue engineering. Transpl Immunol 2002, 9:323-329.

15. Lei $P$, Andreadis ST: Efficient retroviral gene transfer to epidermal stem cells. Methods Mol Biol 2008, 433:367-379.

16. Bajaj B, Behshad S, Andreadis ST: Retroviral gene transfer to human epidermal keratinocytes correlates with integrin expression and is significantly enhanced on fibronectin. Hum Gene Ther 2002, 13:1821-1831.

17. van den Bogaard EH, Rodijk-Olthuis D, Jansen PA, van Vlijmen-Willems IM, van Erp PE, Joosten I, Zeeuwen PL, Schalkwijk J: Rho kinase inhibitor $\mathrm{Y}-27632$ prolongs the life span of adult human keratinocytes, enhances skin equivalent development, and facilitates lentiviral transduction. Tissue Eng Part A 2012, 18:1827-1836.

18. Nanba D, Toki F, Matsushita N, Matsushita S, Higashiyama S, Barrandon Y: Actin filament dynamics impacts keratinocyte stem cell maintenance. EMBO Mol Med 2013, 5:640-653.

19. Rheinwald JG, Green H: Serial cultivation of strains of human epidermal keratinocytes: the formation of keratinizing colonies from single cells. Cell 1975, 6:331-343.

20. Rochat A, Kobayashi K, Barrandon Y: Location of stem cells of human hair follicles by clonal analysis. Cell 1994, 76:1063-1073.

21. Miyoshi H, Blomer U, Takahashi M, Gage FH, Verma IM: Development of a self-inactivating lentivirus vector. J Virol 1998, 72:8150-8157.

22. De Luca M, Tamura RN, Kajiji S, Bondanza S, Rossino P, Cancedda R, Marchisio PC, Quaranta V: Polarized integrin mediates human keratinocyte adhesion to basal lamina. Proc Natl Acad Sci USA 1990, 87:6888-6892.

23. Pellegrini G, Dellambra E, Golisano O, Martinelli E, Fantozzi I, Bondanza S, Ponzin D, McKeon F, De Luca M: p63 identifies keratinocyte stem cells. Proc Natl Acad Sci USA 2001, 98:3156-3161.

24. Senoo M, Pinto F, Crum CP, McKeon F: p63 Is essential for the proliferative potential of stem cells in stratified epithelia. Cell 2007, 129:523-536.

25. Rice $\mathrm{RH}$, Green $\mathrm{H}$ : The cornified envelope of terminally differentiated human epidermal keratinocytes consists of cross-linked protein. Cell 1977, 11:417-422.

26. Goff SP: Intracellular trafficking of retroviral genomes during the early phase of infection: viral exploitation of cellular pathways. J Gene Med 2001, 3:517-528. 
27. Li A, Simmons PJ, Kaur P: Identification and isolation of candidate human keratinocyte stem cells based on cell surface phenotype. Proc Natl Acad Sci USA 1998, 95:3902-3907.

28. McMullan R, Lax S, Robertson VH, Radford DJ, Broad S, Watt FM, Rowles A Croft DR, Olson MF, Hotchin NA: Keratinocyte differentiation is regulated by the Rho and ROCK signaling pathway. Curr Biol 2003, 13:2185-2189.

29. Chapman S, Liu X, Meyers C, Schlegel R, McBride AA: Human keratinocytes are efficiently immortalized by a Rho kinase inhibitor. J Clin Invest 2010, 120:2619-2626.

30. Terunuma A, Limgala RP, Park CJ, Choudhary I, Vogel JC: Efficient procurement of epithelial stem cells from human tissue specimens using a Rho-associated protein kinase inhibitor Y-27632. Tissue Eng Part A 2010, 16:1363-1368.

31. Seitz B, Baktanian E, Gordon EM, Anderson WF, LaBree L, McDonnell PJ: Retroviral vector-mediated gene transfer into keratocytes: in vitro effects of polybrene and protamine sulfate. Graefes Arch Clin Exp Ophthalmol 1998, 236:602-612

32. Lin P, Correa D, Lin Y, Caplan Al: Polybrene inhibits human mesenchymal stem cell proliferation during lentiviral transduction. PLoS One 2011, 6:e23891.

33. Barrandon Y, Grasset N, Zaffalon A, Gorostidi F, Claudinot S, Droz-Georget SL, Nanba D, Rochat A: Capturing epidermal stemness for regenerative medicine. Semin Cell Dev Biol 2012, 23:937-944.

34. Amano M, Nakayama M, Kaibuchi K: Rho-kinase/ROCK: A key regulator of the cytoskeleton and cell polarity. Cytoskeleton (Hoboken) 2010, 67:545-554.

35. Schofield AV, Steel R, Bernard O: Rho-associated coiled-coil kinase (ROCK) protein controls microtubule dynamics in a novel signaling pathway that regulates cell migration. J Biol Chem 2012, 287:43620-43629.

36. Bukrinskaya A, Brichacek B, Mann A, Stevenson M: Establishment of a functional human immunodeficiency virus type 1 (HIV-1) reverse transcription complex involves the cytoskeleton. J Exp Med 1998, 188:2113-2125.

37. Komano J, Miyauchi K, Matsuda Z, Yamamoto N: Inhibiting the Arp2/3 complex limits infection of both intracellular mature vaccinia virus and primate lentiviruses. Mol Biol Cell 2004, 15:5197-5207.

38. McDonald D, Vodicka MA, Lucero G, Svitkina TM, Borisy GG, Emerman M, Hope TJ: Visualization of the intracellular behavior of HIV in living cells. J Cell Biol 2002, 159:441-452.

39. De Luca M, Pellegrini $G$, Green H: Regeneration of squamous epithelia from stem cells of cultured grafts. Regen Med 2006, 1:45-57.

40. Mathor MB, Ferrari G, Dellambra E, Cilli M, Mavilio F, Cancedda R, De Luca M: Clonal analysis of stably transduced human epidermal stem cells in culture. Proc Natl Acad Sci USA 1996, 93:10371-10376.

\section{doi: $10.1186 /$ scrt338}

Cite this article as: Nanba et al.: Efficient expansion of human keratinocyte stem/progenitor cells carrying a transgene with lentiviral vector. Stem Cell Research \& Therapy 2013 4:127.

\section{Submit your next manuscript to BioMed Central and take full advantage of:}

- Convenient online submission

- Thorough peer review

- No space constraints or color figure charges

- Immediate publication on acceptance

- Inclusion in PubMed, CAS, Scopus and Google Scholar

- Research which is freely available for redistribution 